\title{
On the evaluation of storage facilities on the dustiness of the urban environment
}

\author{
Vladislav Lupinogin ${ }^{1, *}$, Valeriy Azarov ${ }^{1}$, Evgeny Gorshkov ${ }^{2}$ and Maksim Nikolenko ${ }^{3}$ \\ ${ }^{1}$ Institute of Architecture and Civil Engineering of Volgograd State Technical University, 1 \\ Akademicheskaya st., Volgograd, 400074, Russia \\ ${ }^{2}$ All-Russian Research Institute of Ecology, Russia \\ ${ }^{3}$ Don State Technical University, 1 Gagarin Square, Rostov-on-Don, 344000, Russia
}

\begin{abstract}
Examined the classification of dust from different storage facilities. Were considered different samples of dust (construction, textile, food), generated in storage facilities. Studied the influence of anti-dust coverage on dustiness of storage facilities as the pollution source. This paper presents the results of chemical analysis, studied dustiness, determined classification of studied dust. Constructed integral distribution curves of mass particles by equivalent diameter.
\end{abstract}

\section{Introduction}

In the production of construction materials, dust is considered to be solid particles, the size of which is less than $150 \mu \mathrm{m}$. The dust particles of construction materials is divided by size on these categories [1]:

1. Large-dispersed dust $(50-150 \mu \mathrm{m})$, which is quickly falls out from the dispersion medium and is noticeable without the use of special devices;

2. Small-dispersed dust $(20-50 \mu \mathrm{m})$, which has a low subsidence speed and can be examined under a microscope at small increases;

3. Fine dust $(0,1-20 \mu \mathrm{m})$, which is difficult to settle out from the dispersion medium and visible in standard microscopes;

4. Ultra-fine dust (less than $0,1 \mu \mathrm{m}$ ), which is in Brownian motion, that can be detected using an electron microscope.

There are also other classifications. Despite their diversity - it can be said, that many authors [2,3] considers dust is small- or fine dispersed with the size not larger than $20 \mu \mathrm{m}$. The characteristics of dust from different dispersion groups differs from each other, both in quantitative changes in the dust physical properties and in the nature of the laws expressing these changes.

\footnotetext{
* Corresponding author: vladlupinogin@gmail.com
} 


\section{Methods}

Currently, in a number of countries, including Russia, the content of particles in the atmosphere is rationed with the sizes not over 2,5 $\mu \mathrm{m}$ (PM2,5) and $10 \mu \mathrm{m}$ (PM10), ultradispersed dust is the most dangerous, because it can get inside of human lungs. A system for monitoring and evaluating the concentration and composition of small sized particles in storage facilities is currently absent, which does not allow to give an objective evaluation of the dust influence on the quality of the working environment and air emissions.

The aim of the research is to improve the evaluation of storages as a source of dust pollution of the environment, to develop, based on the acquired data, measures to improve the ecological safety of these premises.

To achieve this goal the following tasks were solved:

Determination of the dust small fractions concentration of non-production storages (PM10, PM2,5), and also, study the influence of anti-dust coverage on dustiness of the environment near storages;

The storage as the source of pollution has some dust emission zones, through which dust, subsided on the floor and service platforms, released in the environment. These include deflectors, various openings in the room and aspiration systems in the presence of technological operations (packaging, overfilling, etc.).

In order to evaluate the pattern of air pollution near storage facilities, was used the method of determining the concentration and dispersal composition of dust inside and outside storages. Measurements of dispersal composition and concentration of dust were carried out in accordance with normative documents [4-7]. Several points of dust concentration measurements points were chosen. The points were in unloading/loading zone. Also, was considered further distribution of harmful substances from the windward and leeward sides. Since a loading-unloading works zone is unorganized source of pollutants emission, to calculate the dust emission volumes were used the following formulas:

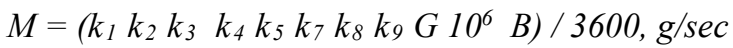

$$
\begin{aligned}
& M=\left(k_{4} k_{5} k_{6} k_{7} q F_{p a \sigma}\right)+\left(\left(k_{4} k_{5} k_{6} k_{7} 0,11 q\left(F_{n л}-F_{\text {раб }}\right)(1-\square)\right)\right. \text {, } \\
& \text { (g/sec), } \\
& M=\left(k_{1} k_{2} k_{3} k_{4} k_{5} k_{7} k_{8} k_{9} G 10^{6} \mathrm{~B}\right) / 3600, \mathrm{~g} / \mathrm{sec}
\end{aligned}
$$

Where the ratios, used for calculations during unloading, storage and loading, are taken from the methodical guide [8]. Further, with the help of the program UPRZA "Ecologist" (Fig. 1) were giving the coordinates for the source of dust emissions from the storage during the loading-unloading works, and also, the coordinates of points at the distance from 50 meters (C33) to 600 meters (end of the residential area) and concentrations were acquired in the points of given coordinates. Based on the acquired data, the distribution picture of the dust emission concentration from the storage in the loading-unloading zone, was constructed (Fig. 2). 


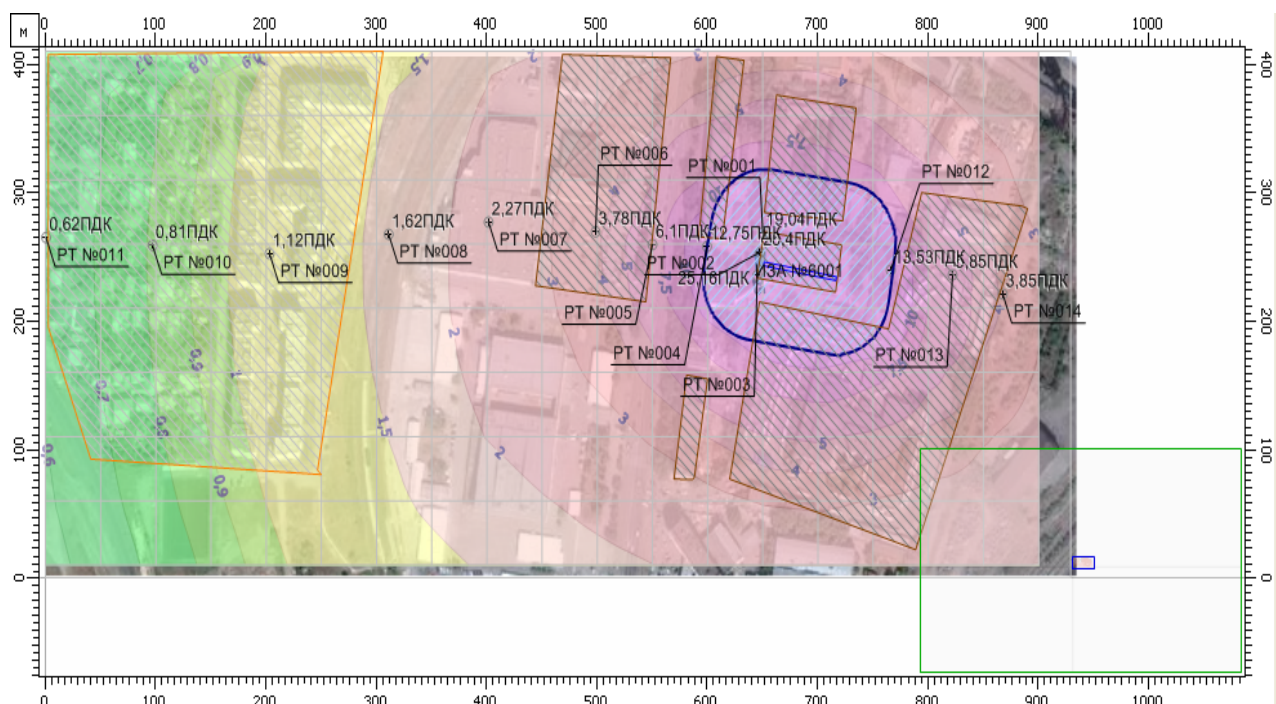

Fig.1. Concentration of dust in different points from the storage facility during the loading-unloading works.

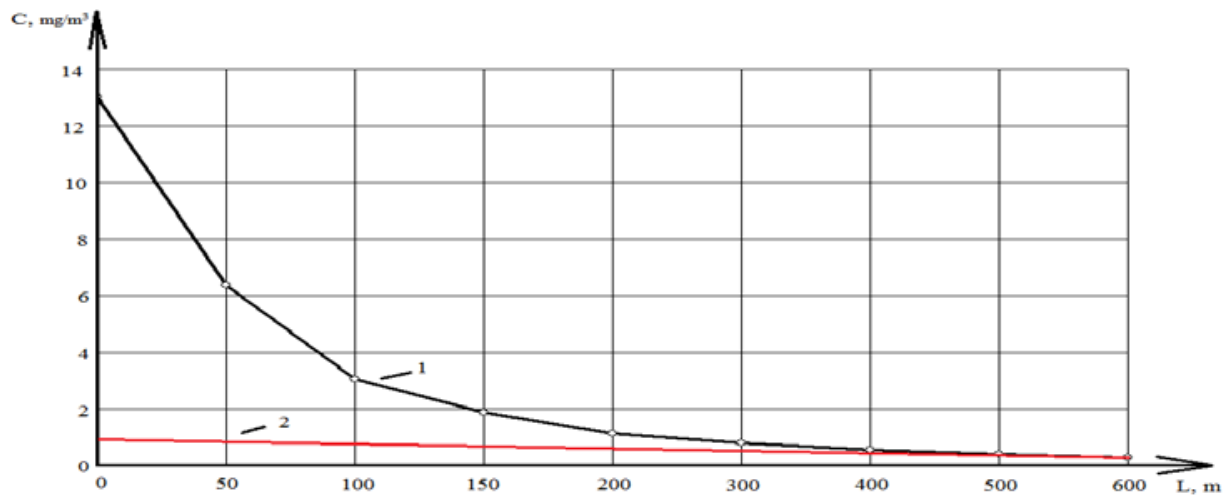

Fig. 2. The dependence of dust concentration from storage facilities on the distance: 1 - from the gates of loading-unloading works; 2 - in the atmosphere of urban environment.

Defined the total and fractional dust concentrations. Constructed integral distribution functions $\mathrm{F}(\delta)$. Then, by values of $\mathrm{F}$ for $\delta=2 \mu \mathrm{m}$ and $\delta=10 \mu \mathrm{m}$ were determined the values for $\mathrm{PM}_{10}$ and $\mathrm{PM}_{2,5}$ :

$$
\begin{aligned}
& P M_{2,5}=(C F(d)) / 100, \% \\
& P M_{10}=(C F(d)) / 100, \%
\end{aligned}
$$

where $C$ - total dust concentration in researched point, $\mathrm{mg} / \mathrm{m}^{3}$,

$P M_{2,5}$ and $P M_{10}$ - concentration of dust with the size less than $2,5 \mu \mathrm{m}$ and $10 \mu \mathrm{m}$, accordingly, $\mathrm{mg} / \mathrm{m}^{3}$,

$F(d)$ - integral distribution function of particles by equivalent diameter, $\%$,

$d=$ equivalent particle diameter [9]

For determination of dispersal composition and characteristics of dust in the air of storage facilities, the microscopy method was used [10-12]. In this regard, was conducted the dust analysis, sampled in the atmosphere from the storages for three types of the materials. On the Fig. 3 presented integral distribution functions of mass dust particles by 
equivalent diameter in the probabilistic-logarithmic coordinate system for storage facilities of textile, construction and food materials, in the lower and upper zones.

According to the data, acquired in the experiment and shown on Fig.3, it may be concluded, that the size of dust particles in the upper zone is a lot smaller, then in the lower zone. For textile dust $\mathrm{d}_{50}=4 \mu \mathrm{m}$ (in the upper zone) and $\mathrm{d}_{50}=5,8 \mu \mathrm{m}$ (in the lower zone); for food dust $\mathrm{d}_{50}=9 \mu \mathrm{m}$ (in the upper zone) и $\mathrm{d}_{50}=18 \mu \mathrm{m}$ (in the lower zone); for construction dust $\mathrm{d}_{50}=6,5 \mu \mathrm{m}$ (in the upper zone); $\mathrm{d}_{50}=10 \mu \mathrm{m}$ (in the lower zone).

In the course of the research, was implemented the chemical analysis of construction materials dust, with the subsequent micro-X-ray analysis. The analysis was performed on optical microscope 3D Versa. With the use of this microscope, were measured the sizes of construction dust particles with different zoom. For comparison were chosen samples of some representative dust samples from the storage. In composition of all samples were found silicates and carbonates of alkali and alkaline earth metals, sodium chloride, iron oxide and molybdenum oxide, and aluminum oxide. Silicon oxide prevails. The composition is close to bauxite, this is confirmed by stored material in the storages - brick. The size of a small particles (Fig. 3) varies from $0,6 \mu \mathrm{m}$ to $0,7 \mu \mathrm{m}$. Such dust is generated, for example, during the technological operations (packing, overfilling, cutting, and etc.). Dust of this size causes significant harm to workers in this room, also, it is taken outside of the storage. Consequently, it becomes necessary to install additional dust collectors.

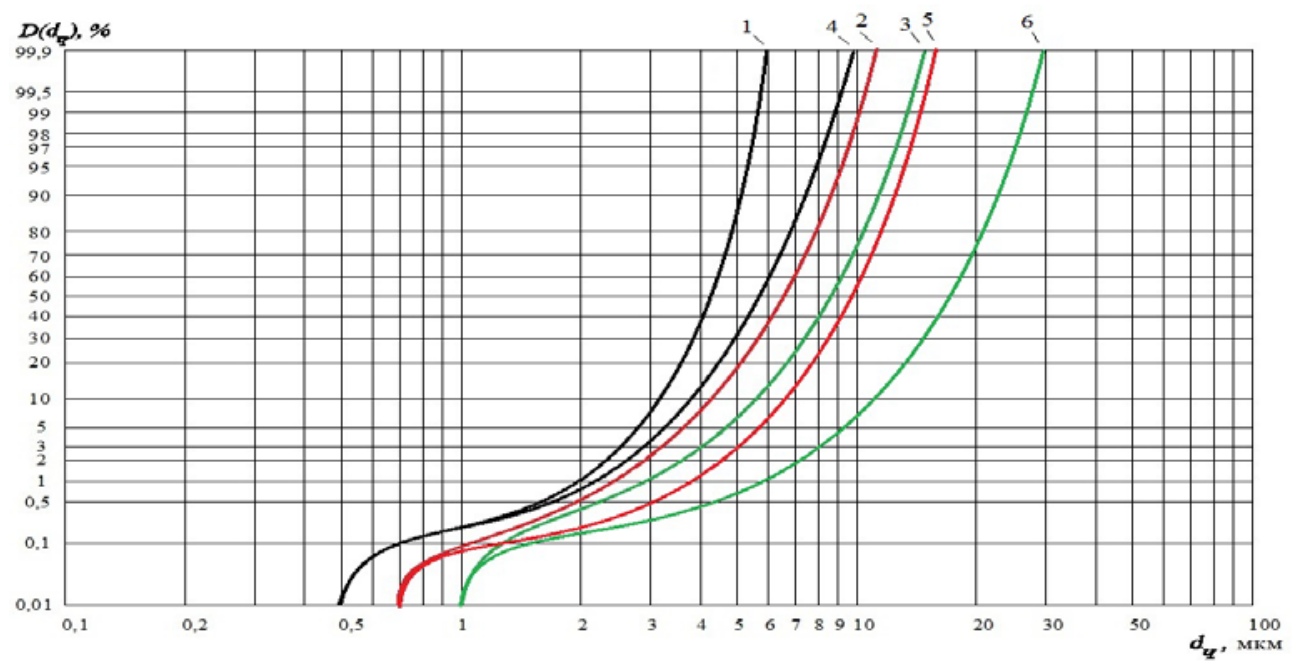

Fig. 3. Integral distribution functions of mass particles by diameter in the upper $(1-$ textile; 2 construction materials; 3 - food) and the lower (4 - textile; 5-construction materials; 6 - food) storage zones

The research, conducted near storages for food, construction and textile, allowed to obtain data on distribution of dispersal dust composition during at different wind directions relatively to the storage (leeward and windward sides), and with an anti-dust coverage, and without it. As an example, the Fig. 4 and 5, show the results of the studies for the distribution function of construction and food dust. According to the acquired data, for both types of storages, in the air from windward side the particles of dust were larger, because, mostly - it is urban and roadside dust. 


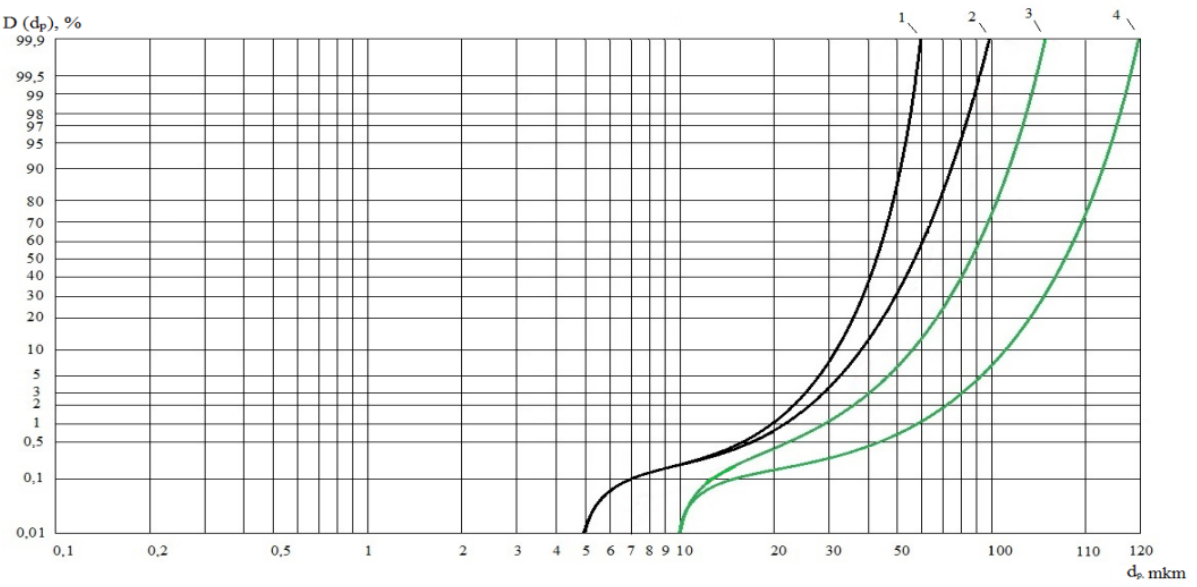

Fig. 4. Integral curves distribution of mass dust particles by equivalent diameter in air of the sanitary protection zone of the storages without anti-dust coverage from the leeward side (1 - construction materials, 3 - food) and from the windward side ( 2 - construction material, 4 - food)

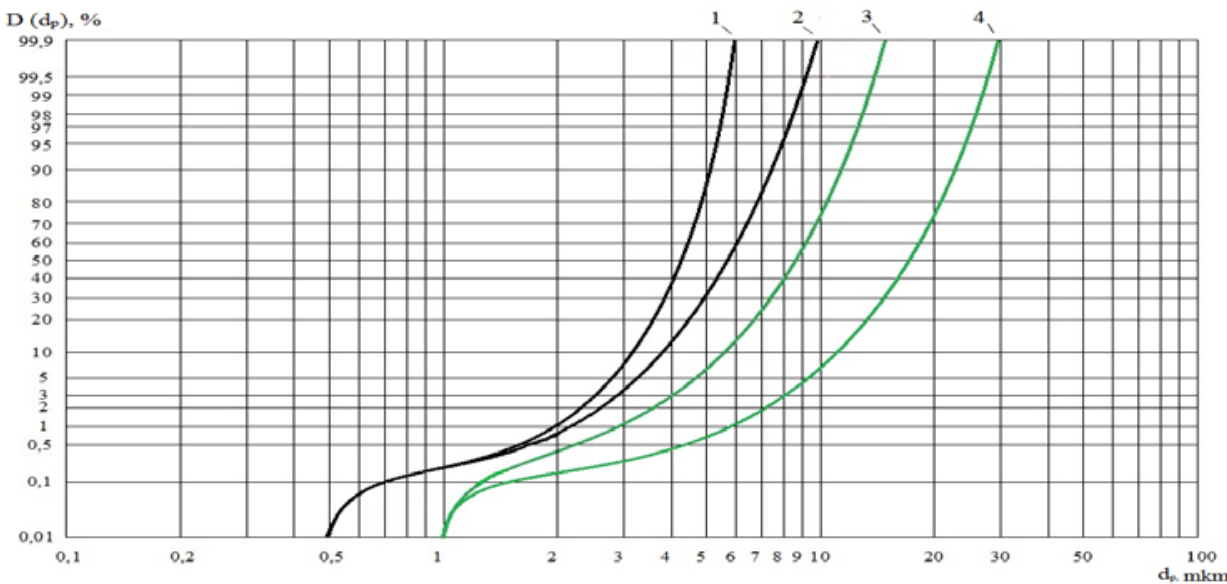

Fig. 5. Integral curves distribution of mass dust particles by equivalent diameter in air of the sanitary protection zone of the storages with anti-dust coverage from the leeward side $(1$ - construction material, 3 - food) and from the windward side (2 - construction material, 4 - food).

\section{Results and Discussion}

Research, conducted for storages with anti-dust coverage in the unloading/loading zone of materials (Fig.5) showed, that in this case the percentage of small-dispersed dust of $\mathrm{PM}_{10}$ drastically increases (to $100 \%$ ). Nevertheless, the total and integral fractional concentration in storages with anti-dust coverage is smaller.

The conducted studies allowed to analyze the ratio of the dust dispersal composition of harmful substances in the air from the leeward and windward sides of the storage, near to the sanitary protection zone with the existence and absence of anti-dust coverage. Nevertheless, the wind with the speed 3-6 m/s was bringing large dust from the windward side (from the sanitary protection zone) of the road. And on the other side (leeward side) small dust. Accordingly, with the existence of anti-dust coverage in storages, smalldispersed dust is more prevalent. The proportion of construction materials on leeward and 
windward sides with anti-dust coverage, with a size of $\mathrm{PM}_{10}$ and less, in the air of the working zone is $100 \%$ from the total mass of dust. The content of dust on the leeward and windward sides without anti-dust coverage, with a size of $\mathrm{PM}_{10}$ and less, in the air of the sanitary protection zone is $0,5 \%$ from the total mass of dust. The content of dust with the size of $\mathrm{PM}_{2,5}$ in the storages without anti-dust coverage is absent. In the storages with antidust coverage on a windward and leeward sides the content of $\mathrm{PM}_{2,5}$ particles are approximately an equal and amounts to $3 \%$ of the total mass of dust.

Similarly, based on the research given by the author, the content of food dust on leeward and windward sides without anti-dust coverage, with a size of $\mathrm{PM}_{10}$ and more in the air of the working zone is $99 \%$ from the total mass of dust, with anti-dust coverage on the windward side is $70 \%$, on the leeward side - $8 \%$. Other - large particles. The content of dust on the leeward and windward sides without anti-dust coverage, with a size of $\mathrm{PM}_{2,5}$ in the air of the sanitary protection zone is absent. The content of dust on the leeward side with anti-dust coverage, with a size of $\mathrm{PM}_{2,5}$ is less than $1 \%$, on the windward side $-0,3 \%$. At high wind speed 6-12 m/s the picture changed. Large-dispersed dust did not settle in the SPZ of the storage, therefore, the concentration at the border of the sanitary zone of the storages increased (to $30 \%$ ), then on the windward. The proportion of small-dispersed dust increased $\mathrm{PM}_{10}$ (to $26 \%$ ). The proportion of $\mathrm{PM}_{2,5}$ in all studies did not exceed $5 \%$.

\section{Summary}

The results of the experiment showed, that the largest percentage of dust removal from the storage depends on the humidity index, wind speed and particle size. Furthermore, was studied the influence of dust removal from storages on the dustiness of the environment.

Dust size depends on the side of the storage relatively to wind and from existence of anti-dust coverage.

In addition to meteorological parameters, the acquired results are also influenced by the density, size and sorbing properties of particles, as a result - the influence of total dust concentration on the content of small fractions in residential area, which are located near the industrial enterprises, requires further study.

The classification of studied dust is determined.

For all types of storages the dust, from the windward side, as a rule, is smaller.

\section{References}

1. E.P. Mednikov, Turbulent transfer and subsidence of aerosols, (M.: Science, 1981)

2. N.A. Fuchs, Mechanics of aerosols (M.: USSR Academy of Sciences, 1955)

3. N.A. Fuchs, A.G. Strugin, High-dispersed aerosols (M., 1969)

4. GOST 17.2.1.04-77 Protection of Nature. Atmosphere. Sources and meteorological factors of pollution, industrial emissions (M.: Standards Publishing, 1978)

5. GOST 17.2.4.02-81 Protection of Nature. Atmosphere. General requirements for methods for the determination of pollutants (M.: Standards Publishing, 1982)

6. RF Law from.05.1999 «On the protection of atmospheric air», № 96-Ф3, 1999.

7. RF Law from 19.12.1991 «On the protection of the environment», Vedomosti SND, 10, (1992)

8. Methods for calculating emissions from unorganized sources in in the building materials industry (Novorossiysk: CJSC «NIPIOSTROM», 2001) 
9. I.V. Stefanenko, V.N. Azarov, K.A. Trohimchuk, M.V. Trohimchuk, Research of air flows dynamics at construction works in the condition of urban built-up areas, Applied Mechanics and Materials. The 2nd International Conference Material, v. 875, pp. 183186 (2018). doi: 10.4028/www.scientific.net/AMM.875.183.

10. V.N. Azarov, S.A. Koshkarev, Increasing the environmental safety of the construction industry by improving dust removal systems, with the usage of a comprehensive dispersion analysis of dust emissions, Bulletin of Institute of Architecture and Civil Engineering of Volgograd Series: Construction and architecture, 43, 161-174 (2016)

11. V.N. Azarov, N.M. Sergina, T. Kondratenko, Problems of the protection of urban ambient air pollution from industrial dust emissions, MATEC Web of Conferences. Vol. 106: International Science Conference SPbWOSCE-2016 «SMART City», (2017) URL:https://www.matec-conferences.org/articles/matecconf/abs/2017/20/contents/ contents.html.

12. V.N. Azarov, A.I. Evtushenko, V.P. Batmanov, A.B. Strelyaeva, V.V. Lupinogin, Aerodynamic Characteristics of Dust in the Emissions into the Atmosphere and Working Zone of Construction Enterprises, International Review of Civil Engineering, v. 7(5), pp. 132-136 (2016) 